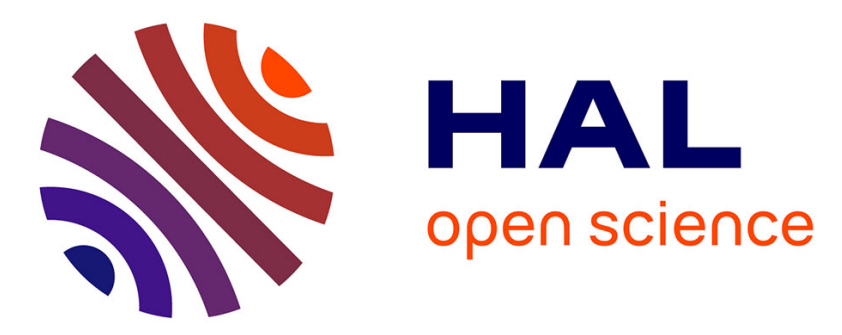

\title{
Design and realization of a microfluidic device devoted to the application of ultra-short pulses of electrical field to living cells
}

Claire Dalmay, Julien Villemejane, Vanessa Joubert, Olivier Français, Lluis M. Mir, Bruno Le Pioufle

\section{To cite this version:}

Claire Dalmay, Julien Villemejane, Vanessa Joubert, Olivier Français, Lluis M. Mir, et al.. Design and realization of a microfluidic device devoted to the application of ultra-short pulses of electrical field to living cells. Sensors and Actuators B: Chemical, 2011, 160 (1), pp.1573-1580. 10.1016/j.snb.2011.09.009 . hal-00738711

\section{HAL Id: hal-00738711 \\ https://hal.science/hal-00738711}

Submitted on 12 Feb 2013

HAL is a multi-disciplinary open access archive for the deposit and dissemination of scientific research documents, whether they are published or not. The documents may come from teaching and research institutions in France or abroad, or from public or private research centers.
L'archive ouverte pluridisciplinaire HAL, est destinée au dépôt et à la diffusion de documents scientifiques de niveau recherche, publiés ou non, émanant des établissements d'enseignement et de recherche français ou étrangers, des laboratoires publics ou privés. 


\title{
Design and realization of a microfluidic device devoted to the application of ultra-short pulses of electrical field to living cells
}

\author{
Claire Dalmay ${ }^{\mathrm{a}}$, Julien Villemejane $e^{\mathrm{a}, \mathrm{b}, \mathrm{c}}$, Vanessa Joubert ${ }^{\mathrm{b}, \mathrm{c}}$, Olivier Français ${ }^{\mathrm{a}}$, Lluis M. \\ Mir $^{\mathrm{b}, \mathrm{c}}$, Bruno Le Pioufle ${ }^{\mathrm{a}^{*}}$
}

${ }^{a}$ SATIE UMR CNRS 8029, Institut d'Alembert, ENS Cachan, 61 av President Wilson, F-94235 Cachan, France

${ }^{b}$ UMR CNRS 8203, Institut Gustave-Roussy, 114 rue Edouard. Vaillant, F-94805 Villejuif, France

${ }^{c}$ Univ Paris-Sud, F-94805 Villejuif, France

*corresponding author : e-mail: bruno.lepioufle@satie.ens-cachan.fr, Tel: +3314740 77 36, Fax: +3314740 2065

\begin{abstract}
In this paper, we present a novel microfluidic system dedicated to the application of ultra short pulses (i.e. nanopulses) on cells and the visualization of their effects. Cell plasma membranes can be rendered permeable by the use of nanosecond pulsed electric field. In conventional macroscopic electroporation chambers, the typical pulse duration is on the order of several hundred microseconds (micropulses), which allows the penetration of genes or other therapeutic molecules inside the cell. Consequently, electroporation pulses can be used in various clinical applications such as skin cancer treatment. A huge interest recently appeared on the use of ultra-short electric pulses (few nanoseconds) for the treatment of cells, as it was shown to enhance the treatment efficacy compared to micropulses. In this paper, the design, fabrication and biological validation of a microfluidic biochip optimized for the nanoporation of cells is detailed. The developed system presents several advantages such as real time monitoring of the effects of nanoporation on cells, single cell analysis, local generation of extremely high strength electrical fields while using reasonably low voltages and the precise control of electrical and fluidic parameters. To achieve the required spatial homogeneity of the electric field within the fluidic channel, the biochip includes thick electroplated gold electrodes ( $\mathrm{t}=6$ $30 \mu \mathrm{m})$. As demonstrated in the paper, the electrical connections between the chip and the nsPEF generator are of prime importance, as it affects directly the quality of the impedance matching i.e. the transfert of energy between the generator and the cells. A study of the effects of these electrical connections on the biochip impedance is detailled. Nanoporation was achieved on living cells within the proposed chip, and characterized through the measurement of the fluorescence decay of intracellular calcein.
\end{abstract}

Keywords : Nanoporation, biochip, biological cells, electroplating.

\section{Introduction}

The application of electric pulses is a well-known method to induce permeabilization of the cell plasma membrane that permits the penetration of genes or drugs into the cytosol of cells. Typical electropermeabilization methods require pulses of about $1 \mathrm{kV} / \mathrm{cm}$ and duration of $100 \mu \mathrm{s}$. They are currently used for electrochemotherapy [1,2], to treat cutaneous and subcutaneous tumour nodules, to potentiate the cytotoxicity of nonpermeant or poorly permeant anticancer drugs, or in gene electrotransfer to various animal tissues [3,4,5]. More recently electroporation has been used on human tissues in the framework of clinical trials [6]. A new complementary technique - called nanoporation - is emerging, based on the exposure of cells to ultrashort pulsed electric fields - nanosecond pulsed electric fields (nsPEF) - which fall in the range of 4 to $300 \mathrm{~ns}$ duration, and amplitudes of 20 to $150 \mathrm{kV} / \mathrm{cm}$. These kinds of pulses are very promising. They allow for the electropermeabilization of the plasma membrane for small molecules $[7,8]$, but also permit to shunt the insulating effect of the plasma membrane and so they are capable to act on the intracellular component of cells, like the endoplasmic reticulum [9] or the mitochondria [10]. Nevertheless, both effects on the plasma membrane and intracellular components are misunderstood. The purpose of the presented work is to take benefit of microtechnologies to develop a biochip to investigate the effects of these kinds of pulses. In this paper we investigate the effect of nsPEF on the plasma membrane, as this step can be achieved using classical fluorescent dies.

Commonly, to apply pulsed electric field to cells, commercial electroporation chambers, with large and distant 
aluminum electrodes $(\mathrm{d}=1-2 \mathrm{~mm})$, are commonly used in laboratories to investigate their effect on living cells but present some drawbacks. Microfluidic systems are advantageous compared to these macroscopic chambers, as they allow a precise spatial and temporal control of electrical parameters $[11,12]$. They permit to achieve a homogeneous electrical field [13] and $\mathrm{pH}$, to reduce electrochemical effects and sample contamination and analysis volumes $[14,15]$. In addition, these microsystems also allow for the development of different solution for the real time monitoring of cells during the application of pulses without decreasing of the cell viability [16]. Possibility of cells

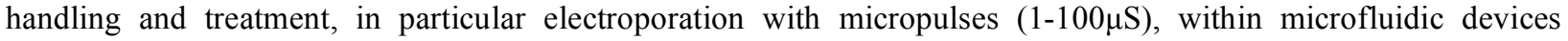
manipulating droplets is reported in the literature [17]. Same type of electroporation applied to cells flowing between thick electrodes is also described in other works. In particular [18] reports a system where an aluminum bar ( $0.5 \mathrm{~mm}$ thick) embedded in a PMMA plate was milled to define a $2 \mathrm{~mm}$ wide channel.

Within this context, we propose the development of a new microfluidic device, specially designed for the application of ultrashort pulses (nanopulses) to cells flowing between thick electrodes. Our device involves thick electrodes as a flowing channel for cells that are electroporated. At the difference with [18] miniaturized gold electrodes are employed within our device. Indeed gold ensures biocompatibility of the chip, while miniaturization is necessary to apply the extremely high electrical field that we intend to apply. Gold is electroplated prior to chemical etching following a design defined by photolithography in order to achieve the necessary miniaturization, with the proper impedance matching, considering the high frequency spectrum of the applied electrical pulses.

Hence, the microfluidic device that we propose in this paper has been optimized for the application of nsPEF on living cells and permits their real time monitoring. The design and fabrication of the biochip is described in the next section. As the impedance matching is crucial for an optimal transfer of the nsPEF energy towards the living cells exposed within the microfluidic chip, a characterization of the impedance of the system was made. The effects of the electrical connections used between the biochip and the generator were taken into account in the study. Finally the feasibility to use the proposed microfluidic chip to achieve nanoporation was demonstrated through the real time observation of the fluorescence decay of cells previously charged with intracellular calcein, and exposed to nsPEF.

\section{Materials and Methods}

\subsection{Design and simulation}

In order to guarantee a sufficient spatial uniformity of the electric field applied to the cells which are flowing within the microchannel, the thickness of electrodes has to be at least higher than the cell radius $\left(\mathrm{e}_{\mathrm{el}}>6 \mu \mathrm{m}\right)$. This led us to develop a device with thick electroplated electrodes (thickness from $6 \mu \mathrm{m}$ up to $25 \mu \mathrm{m}$, to compare with the thickness of electrodes obtained through metal evaporation $t \sim 150 \mathrm{~nm}$ ). It is of prime importance to apply an homogeneous and well controlled electrical field to the biological cells flowing in the microfluidic channel to ensure that flowing cells see the same level of electrical field, whatever their position in the section of the channel, and are thus electropermeabilized with the same conditions.

Gold is chosen as material for the electrodes to ensure biocompatibility. The microchannel is defined by the thick gold electrodes, to which is superposed a patterned SU8 layer in order to obtain the desired channel height. The device is packaged using two different ways i) a microscope glass slide ( $\mathrm{t}=170 \mu \mathrm{m})$, simply covering the chip ii) a PDMS layer, covalently bonded to the surface of SU8 structure (Fig. 1). Fluidic inlets and outlets are created by drilling the glass slide or punching the PDMS cover.

In order to expose biological cells to nsPEF and to monitor them in the microfluidic chip, two main constraints appear: (i) matching of the biochip impedance with the pulse generator impedance (set to 50 ohms), in order to deliver a maximum of energy to the cell medium while avoiding pulse reflections which might damage the RF generator (ii) planarity, parallelism and transparency between the different layers of the chip (Fig. 1) for microscopic observations of the nanoporation experiments.

\section{Insert Figure 1}

Metallic electrodes (Fig. 2) are designed in such a way that the pulsed electric field which penetrates the chip is mainly dissipated within the biological medium (which typical conductivity $\sigma_{\mathrm{m}}$ is $1 \mathrm{~S} / \mathrm{m}$ ). If we consider that the 
thickness of electrodes $\left(e_{\mathrm{el}}\right)$ is equal to the microchannel height $\left(\mathrm{h}_{\mathrm{c}}\right)$, and the microchannel width $\left(\mathrm{w}_{\mathrm{m}}\right)$ is equal to the gap between the electrodes $\left(\mathrm{g}_{\mathrm{c}}\right)$, the electric behaviour of the biochip can be seen as a propagation line as long as r.G. $L^{2}<<1$, where $r$ and $G$ stands respectively for the gold electrode linear resistance and the linear medium conductance whereas $\mathrm{L}$ is the length of the channel:

$$
r=\frac{\rho_{e l}}{e_{e l} \cdot w_{e}} ; G=\frac{\sigma_{m} \cdot h_{c}}{g_{c}}
$$

$\mathrm{e}_{\mathrm{el}}$ represents the thickness of the electrodes, $\mathrm{w}_{\mathrm{e}}$ their width and $\rho_{\mathrm{el}}$ their resistivity. $\mathrm{h}_{\mathrm{c}}$ and $\mathrm{g}_{\mathrm{c}}$ are respectively the height and width of the microchannel and $\sigma_{\mathrm{m}}$ is the conductivity the biological medium.

$\mathrm{L}$ (length of the microchannel), is adjusted in order to obtain an impedance matching $\left(\mathrm{Z}_{\mathrm{m}}\right)$ of 50 Ohms. In our configuration, the biological medium mainly influences the impedance of the biochip, as the intrinsic resistance of the electrodes can be neglected, considering their thickness. Thus, if the electrical field frequency spectrum is low enough to neglect the polarization of the medium $\left(\mathrm{f}_{\mathrm{t}}<<\sigma_{\mathrm{m}} / 2 \pi \varepsilon_{\mathrm{m}}\right)$, and if we assume that the electrical field is spatially uniform within the channel, the cell culture medium impedance $Z_{m}$ is:

$$
Z_{m}=\frac{1}{G . L}
$$

Based on these considerations, different configurations have been investigated for the architecture of the microfluidic chip, playing with the different geometrical parameters of the design, in particular with the thickness of electrodes. Indeed, for a given gap $\mathrm{g}_{\mathrm{c}}$, between electrodes, and a given height of the channel $\mathrm{h}_{\mathrm{c}}$, the length of electrodes L is obtained from the formula (2). L is finally adjusted using finite element simulations (Comsol Multiphysics (C), in order to take into account the electric field variations at the vicinity of the electrodes and actual measured dimensions of the chip. Fig. 2 shows two different designs depicted in table 1, which both match to 50 Ohms when the two microfluidic channels are filled with the biological media.

\section{Insert Figure 2 \\ Insert Table 1}

Figure 3 shows results of finite element analysis of the electric field distribution inside the microfluidic channel. We considered four cases for this simulation: (1) electrodes (thickness $e_{e l}$ ) define the microchannel height $h_{c}$, (2) electrodes are thinner $\left(\mathrm{e}_{\mathrm{el}}=20 \mu \mathrm{m}\right)$, and covered with SU8 in order to define the whole height of the microchannel $\left(\mathrm{h}_{\mathrm{c}}=50 \mu \mathrm{m}\right)$. A recess is introduced for the SU8 channel which becomes larger $\left(\mathrm{w}_{\mathrm{m}}=300 \mu \mathrm{m}\right)$ than the gap between electrodes. This second case corresponds to the actual geometry of the biochip and takes into account the constraints of the fabrication process (thickness of electrode, alignment capability between metallic and resist layers). Case (3) corresponds to case (2) but without the recess of SU8. And case (4) corresponds to the case of thin electrodes (t 150nm).

\section{Insert Figure 3}

As illustrated on Figure 3.a, a good homogeneity of the electric field is achieved within the channel in most of cases. Nevertheless peaks on the electrical field are observed at the vicinity of the edges of electrodes (cases (2), (3) and (4)). As shown on Figure 3.b, representing the electrical field level across the microfluidic channel (x position), this edge effect is more important in the case where thin electrodes are used, as strong inhomogeneities appear in the electrical field, resulting in a very inhomogeneous electropermeabilization. In particular, cells are not guided between the electrodes and can be exposed to high electrical fields above or at the border of electrodes [19]. As a consequence, thick electrodes were chosen instead of thin electrodes for our application.

In Figure 3.a, the electrical field in the central region of the channel shows to be slightly different in the four cases (a 14\% decrease of the electrical field is observed in case (3) while this decrease is limited to $6 \%$ in case (2), and reaches $18 \%$ in case (4) compared to the ideal case (1). These variations affect the impedance matching, which is prime importance for the design, and therefore must be taken into account. 


\subsection{Fabrication process of the microfluidic biochip}

A glass substrate was used as the main substrate to ensure transparent and planar surfaces. The material used for the metallic electrodes was gold because of its good conductivity $\left(\sigma=4.1 \times 10^{7} \mathrm{~S} / \mathrm{m}\right)$ and its good biocompatibility. A transparent and biocompatible photoresist SU8 (MicroChem) was used to pattern the microfluidic channel and increase its thickness up to $50 \mu \mathrm{m}$.

We followed the process described here after for the microfabrication of the chip:

(1) Starting with a glass substrate metalized with a $10 \mathrm{~nm} \mathrm{Cr} / 150 \mathrm{~nm} \mathrm{Au}$, we proceeded to the electroplating of a thick gold layer (typically $6-25 \mu \mathrm{m}$ ) covering the whole substrate. The electrolytic solution was based on potassium aurocyanure $(\mathrm{KAu}(\mathrm{CN}) 2)$, the counter electrode was made in platinized titanium. The applied current during the electrodeposition was $30 \mathrm{~mA}$ during cycles of $20 \mathrm{~min}$.

(2) Photosensitive resist (S1805, MicroChem) was spincoated and patterned thanks to UV photolithography, to define the mask for the etching of the gold electrodes.

(3) Wet etching of both electrodeposited gold and seed layers was then achieved.

(4) Removal of the photoresist and cleaning of the wafer were done with successive acetone, isopropyl alcohol and water baths. The planarity of the electroplated electrodes was checked using profilometer measurements (DekTak). The different profile measurements showed an excellent planarity of the electrodes (1.2 $\mu \mathrm{m} \mathrm{level}$ variation over the whole 2 inches wafers).

(5) SU8 (MicroChem) photosensitive thick resist was then patterned to define the microfluidic channel.

(6) Finally, two methods were used for the packaging of the microfluidic chip: i) for the electrical tests (presented section 2.3) a PDMS cover was bonded to the top of the SU8 layer, after silanization, ii) for the biological tests (presented in section 2.4) a glass slide was used to cover the chip during the experimentations, that could be removed for its cleaning.

The silanization process used to bond the SU8 layer with the PDMS cover, involved three main steps: i) oxygen plasma was first used to activate the SU8 surface. ii) Then the sample was immersed in a (3Aminopropyl)trimethoxysilane (APTMS, Aldrich) solution during 30 minutes. iii) After rinsing in methanol and water bath, the sample was placed in a dry oven during 3 hours at $110^{\circ} \mathrm{C}$. Once the silanization of the SU8 layer was achieved, a PDMS layer, activated thanks to oxygen plasma could be irreversibly bonded.

Figure 4 shows photographs of fabricated biochips.

\section{Insert Figure 4}

\subsection{Set-up for the measurement of the influence of the electrical connection}

The influence of the method used to connect the microfluidic chip to the electrical pulse generator has been investigated. The chip we used for this work was the design 1 (table 1), with a packaging performed with a PDMS cover bonded thanks to the silanization of the SU8 layer as explained previously.

As shown on figure 5, two configurations of the electrical connections were compared: the biochip was connected to the generator thanks to short welded wires or using SMA (SubMiniature version A) connectors (Emerson Network Power).

A network analyzer (Hewlett Packard 4195A) connected to an impedance test adapter (Hewlett Packard 4195161001) was used to measure the impedance of the microsystems, involving these connections. This analyzer provides a frequency range between $10 \mathrm{~Hz}$ to $500 \mathrm{MHz}$ which fits well with the bandwidth of the frequency spectrum of the $10 \mathrm{nS}$ duration nanopulses. 


\subsection{Set-up for the biological experiments}

Biological experiments were carried out with DC-3F cells (Chinese hamster fibroblast lung cells). The cells were grown attached in Minimum Essential Medium (Invitrogen, Cergy-Pontoise, France) supplemented with $10 \%$ fetal bovine serum (Invitrogen), $500 \mathrm{U} / \mathrm{ml}$ penicillin, $500 \mu \mathrm{g} / \mathrm{ml}$ streptomycin (Invitrogen) defined as complete medium. Cultures were maintained in a humidified atmosphere with $5 \% \mathrm{CO} 2$ at $37^{\circ} \mathrm{C}$. Cells were routinely passed every two days and kept in culture 24 hours before the nanoporation experiments. One hour before the application of $\mathrm{nsPEF}$, cells were loaded with a fluorescent dye named calcein (through 1 hour incubation with calcein-AM $(1 \mu \mathrm{M})$ at $\left.37^{\circ} \mathrm{C}\right)$.

First experiments were lead with cells maintained in static conditions. Cells were rinsed in PBS (Phosphate Buffered Saline) and detached using Trypsin (Invitrogen). After centrifugation, the cell pellet was suspended in S-MEM solution (typical concentration of cells around $2 \times 10^{5} / \mathrm{ml}$ ). The cell suspension was deposited between electrodes using a micro-syringe and a glass slide was used to cover the system as explained previously. Cells were then exposed to the nanopulses. They were provided by a HV generator (FPG 10-1SM10, FID Technology, Germany) which allows delivering $10 \mathrm{~ns}$ duration pulses with adjustable amplitude up to $10 \mathrm{kV}$.

To highlight the effects of nanosecond pulses on cell membranes, we used calcein-loaded DC-3F cells and we monitored the decay of the intracellular calcein fluorescence caused by the application of the nsPEF. Indeed, this method might be more sensitive compared to commonly used procedures based on an influx of a fluorescent dye in the permeabilized cell as explained in [20]. To visualize the effects on cells, an epifluorescence microscope (Zeiss) was used to follow the evolution of the fluorescence of the calcein in the cells. Before the exposure, one picture was acquired in order to know the initial fluorescence level. After the exposure, one picture per minute was acquired during $20 \mathrm{~min}$.

A negative control was performed showing that cells fluorescence level was constant when none exposed to nsPEF.

Cells viability was finally checked using the Trypan blue $(0.2 \%)$. Trypan blue is a dye that stains in blue the permeabilized or dead cells [21]. In order to observe the proportion of viable cells, Trypan blue was injected below the glass cover slide (by capillary effect) 40 minutes after the exposition of cells to nsPEF. Then it diffused to cells across the microfluidic channel height (below $10 \mathrm{~s}$ for the diffusion time considering the $30 \mu \mathrm{m}$ thick channel).

\section{Results and Discussion}

\subsection{Impedance measurements}

Even if the electrical behaviour of the biochip is mainly governed by the medium properties it can be notably influenced by its electrical connection due to the high frequency components of the nanopulses. Indeed, in order to guarantee an acceptable impedance matching between the biochip and the pulse generator, the design of the biochip as well as its electrical connection has to be optimized carefully for a frequency range fitting with the spectrum of the nanopulse. It is therefore crucial to characterise precisely the energy spectrum of the pulse in order to apply a major part of this energy to the cells inside the microfluidic channel.

Figure 6 shows the frequency spectrum of the applied $10 \mathrm{~ns}$ duration nanopulses, which is mostly localised below $100 \mathrm{MHz}$. In this frequency range $(0,100 \mathrm{MHz})$, the biochip behaviour is purely resistive and linked to the cell culture medium impedance $\mathrm{Z}_{\mathrm{m}}$, which is set to $50 \mathrm{Ohms}$ to match with the impedance of the generator. Consequently the impedance matching is ensured where the maximum of energy of the pulse is concentrated as shown on Figure 6.

For frequencies above $200 \mathrm{MHz}$, the polarisation of the biological medium appears and a transition towards capacitance behaviour occurs. This transition frequency $f_{t}$ depends on both the medium conductivity $\sigma_{m}$ and its relative permittivity $\varepsilon_{\mathrm{r}}(3)$. The measured transition frequency was $200 \mathrm{MHz}$, and was thus lower than the one calculated in formula (3) with the known values for $\sigma_{\mathrm{m}}$ and $\varepsilon_{\mathrm{r}}\left(\sigma_{\mathrm{m}}=1 \mathrm{~S} / \mathrm{m}, \varepsilon_{\mathrm{r}}=78\right)$. This fact is due to the parasitic capacitance introduced by the substrate capacitance.

$$
f_{t}=\frac{\sigma}{2 \pi \varepsilon_{0} \varepsilon_{r}}=231 \mathrm{MHz}
$$

For frequencies above $300 \mathrm{MHz}$, phenomena associated with the electrical connection of the microfluidic chip appear. In the case where the chip was connected with short welded wires, a parallel resonance occurs at $400 \mathrm{MHz}$, as shown in figure 6 . This phenomenon, mainly due to the line inductance effect, disappears in the case where SMA connectors were used instead of wires. 
In our case, the parallel resonant frequency has no consequence considering the nanopulse frequency spectrum (and wire connections are thus acceptable). Nevertheless in the case of the use of shorter pulses (pulse duration below $3 \mathrm{~ns}$ ), the effect of this phenomenon becomes preponderant. Indeed the type of electrical connections of the biochip is of prime importance, as it affects directly the impedance of the system, and consequently the temporal shape of the electrical field pulse applied to the cells flowing within the microfluidic channel.

\section{Insert Figure 6}

\subsection{Real-time observation of nPEF effects on the chip - biological validation of the proposed device}

Cells were charged with calcein as depicted in section 2.4, and flowed within the microfluidic channel. Once the cells were stabilized within the channel, a train of $171 \mathrm{nsPEF}, 10 \mathrm{~ns}$ duration was applied with a repetition frequency of $234 \mathrm{~Hz}$. Then, a train of $99 \mathrm{nsPEF}, 10 \mathrm{~ns}$ duration was applied with a repetition frequency of $10 \mathrm{~Hz}$. Applied voltage $(350$ and $700 \mathrm{~V})$, number and frequency of pulses were fixed by the generator and were not optimized for these first experiments.

\section{Insert Figure 7 \\ Insert Figure 8}

The level of fluorescence was characterized along a cross section in the microfluidic channel (considering the different positions along the $\mathrm{x}$ axis) and monitored versus the time. This gives a 3D plot shown on Figure 7 and 8 were the fluorescence level is represented versus both time and position along $\mathrm{x}$ axis.

As shown in Figure 7.a. and 7.c., we could detect a 60\% decay of the fluorescence level 20 minutes after the application of the nsPEF with an applied voltage of $700 \mathrm{~V}$, demonstrating that a release of the calcein from the cells occurred. This phenomenon was not observed on cells that were not exposed to nsPEF (negative test, Fig.7.b). On figures $7 \mathrm{~b}$ and $7 \mathrm{c}$ the measured fluorescence is represented versus the position in the cross section of the channel (x position), and the time ( $\mathrm{t}$. The initial level of fluorescence at a given position $\mathrm{x}$ is dependant on the number of cells which are at the vicinity of this position in the microfluidic channel. Thanks to the transparency of the device, the cells could be monitored while maintained at the same position $\mathrm{x}$ in the microfluidic channel during the whole recording time. Fig. 8.a. and 8.b show that the application of the nsPEF with an applied voltage of $350 \mathrm{~V}$ allows detecting a 30\% decay of the fluorescent level 20 minutes after the application of the nsPEF.

Complementary experiments of nanoporation with Propidium Iodide are described in another paper of our group [22] and confirm these results.

Finally the viability of cells submitted to nsPEF treatment was confirmed using the Trypan blue test as depicted in section 2.4 with around $97-99 \%$ of exposed cells remaining still alive after being exposed to nsPEF.

These preliminary results demonstrate the feasibility to use our microfluidic biochip for the real-time monitoring of the nanoporation of living cells, and in particular the resulting effects on the plasmid membrane. Based on these results, new biological experiments have to be conducted to optimize the conditions of application and examine other effects of these kinds of pulses on cells.

\section{Conclusion}

First prototypes of biochips for the application of nsPEF on living cells, and real time visualization of their effect were realized and presented in this paper. The miniaturization of the cell exposure chambers allows investigating more intense field strength by reducing the gap between the electrodes. The full transparency of our microfabricated devices allows the observation of the effects of nanopulses on cells. The use of thick electrodes allows the 
application of a very homogeneous electric field to cells flowing within the microfluidic chip. A method permitting to achieve high planarity of these thick electroplated electrodes was proposed. The paper also demonstrates the impact of the electrical connections between the microfluidic chip and the pulse generator, on the capability to transmit the energy spectrum of the nsPEF towards the cells in the exposition channel. A parallel resonance can affect the transmission of the nsPEF especially in the case of ultra-sort pulses with duration below $3 \mathrm{~ns}$.

Biological experiments including real time monitoring of the nanoporation of living cells were carried out using the microfabricated chips. The feasibility of using the proposed chips was characterized thanks to the measurement of the decay of fluorescence of cells charged with calcein prior to the application of nanopulses within the microfluidic channels.

This study constitutes a major step towards the possibility to use microfluidic chips for the real time monitoring of the effects of ultra-short and high amplitude pulsed electric fields on living cells. Advantages of these types of microfluidic devices, discussed in the paper, will be used for further investigations on the effects of nsPEF on intracellular components of living cells.

\section{AKWNOWLEDGMENT}

This work was supported by grants from French National Agency through Nanoscience and Nanotechnology Program (Nanopulsebiochip nANR-08-NANO-024), CNRS, Institut Gustave Roussy, University Paris-Sud, ENS Cachan, PRES Universud and CNANO'Ile de France. 


\section{References}

[1] L.M. Mir, O. Tounekti, S. Orlowski, Bleomycin : revival of an old drug, General Pharmacol. 27 (1996) $745-748$.

[2] M. Marty, et al., Electrochemotherapy - An easy, highly effective and safe treatment of cutaneous and subcutaneous metastases: Results of ESOPE (European Standard Operating Procedures of Electrochemotherapy) study, Eur. J. Cancer. Suppl. 4 (2006) 3-13.

[3] F.M. André et al., Efficiency of high and low voltage pulse combinations for gene electrotransfer in muscle, liver, tumor and skin, Hum. Gene Ther. 19 (2008) 1261-1271.

[4] F.M. André, L.M. Mir, Nucleic acids electrotransfer in vivo: Mechanisms and Practical aspects, Current Gene Therapy 4 (2010) 267-280.

[5] L.M. Mir, Nucleic acids electrotransfer-based gene therapy (electrogenetherapy): past, current, and future, Molecular Biotechnology 43 (2009) 167-176.

[6] L.C. Heller, R. Heller, In vivo electroporation for gene therapy, Hum. Gene Ther 17 (2006) 890-897.

[7] B.L. Ibey, S. Xiao, K.H. Schoenbach, M.R. Murphy, A.G. Pakhomov, Plasma membrane permeabilization by 60- and 60-ns electric pulses is determined by the absorbed dose, Bioelectromagnetics 30 (2009) 92-99.

[8] P.T. Vernier, Y. Sun, M.A. Gundersen, Nanoelectropulse-driven membrane perturbation and small molecule permeabilization, BMC Cell Biol. 7 (2006) 37-53.

[9] S.S. Scarlett, J.A. White, P.F. Blackmore, K.H. Schoenbach, J.F. Kolb, Regulation of intracellular calcium concentration by nanosecond pulsed electric fields, Biochimica Biophysica Acta 1788 (2009) 1168-1175.

[10] S.A. Susin, N. Zamzami, G. Kroener, Mitochondria as regulators of apoptosis : doubt no more, BBABioenergetics 1366 (1998) 151-165.

[11] M. Khine, C. Ionescu-Zanetti, A. Blatz, L.P. Wang, L.P. Lee, Single-cell electroporation arrays with real-time monitoring and feedback control, Lab Chip 7 (2007) 457- 462.

[12] V. Raffa, G. Ciofani, O. Vittorio, V. Pensabene, A. Cuschieri, Carbon nanotube-enhanced cell electropermeabilisation, Bioelectrochemistry 79 (2010) 136-141.

[13] H.Y. Wang, C. Lu, Electroporation of mammalian cells in a microfluidic channel with geometric variation, Anal. Chem. 78 (2006) 5158-5164.

[14] W.G. Lee, U. Demirci, A. Khademhosseini, Microscale electroporation: challenges and perspectives for clinical applications, Integr. Biol. 1 (2009) 242-251.

[15] J.A. Kim, K. Cho,M.S. Shin, W.G. Lee, N. Jung, C. Chung, J.K. Chang, A novel electroporation method using a capillary and wire-type electrode, Biosens. Bioelectron. 23 (2008) 1353-1360.

[16] P.M. Ghosh, C.R. Keese, I. Giaever, Monitoring electropermeabilization in the plasma membrane of adherent mammalian cells, Biophys J. 64(5) (1993) 1602-1609.

[17] Y. Zhan, J. Wang, N. Bao, C. Lu, Electroporation of cells in microfluidic droplets, Anal. Chem. 81 (2009) 2027-2031.

[18] S. Wang, X. Zhang, W. Wang, L. James Lee, Semicontinuous flow electroporation chip for high-throughput transfection on mammalian cells, Anal. Chem. 81 (2009) 4414-4421. 
[19] M. Chiral, M. Denoual, A. Hoel, J. Aguinet, B. Le Pioufle, Electrotransfection results on Jurkat cells on a microdevice, Angioskin, Electroporation based technologies and treatment workshop, (2005).

[20] P.T. Vernier, Z.A. Levine, Y.H. Wu, V. Joubert, M.J. Ziegler, L.M. Mir, D.P. Tieleman, Electroporation fields target oxidatively damaged areas in the cell membrane, Plos One 411 (2009) e7966.

[21] J.R. Tennant, Evaluation of the trypan blue technique for determination of cell viability, Transplantation 2 (1964) 685-694.

[22] C. Dalmay, J. Villemejane, V. Joubert, A. Silve, D. Arnaud-Cormos, O. Français, L.M. Mir, P. Leveque, B. Le Pioufle., Bios. Bioel. (2011) doi:10.1016/j.bios.2011.03.020. 
Figure captions

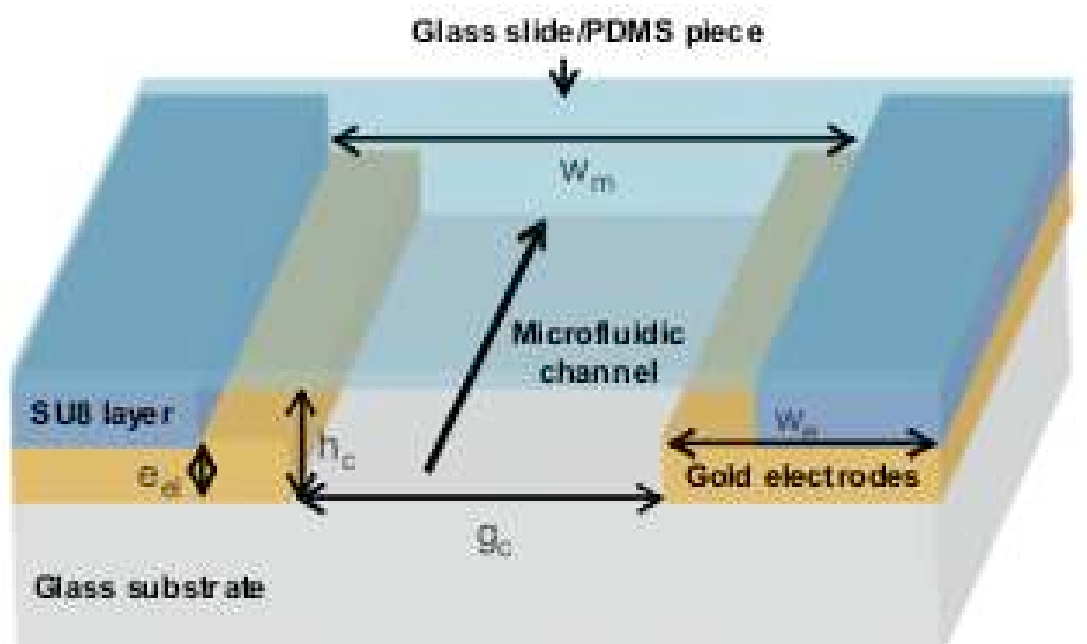

Fig. 1. Schematic cross section of the biochip (arbitrary scale).

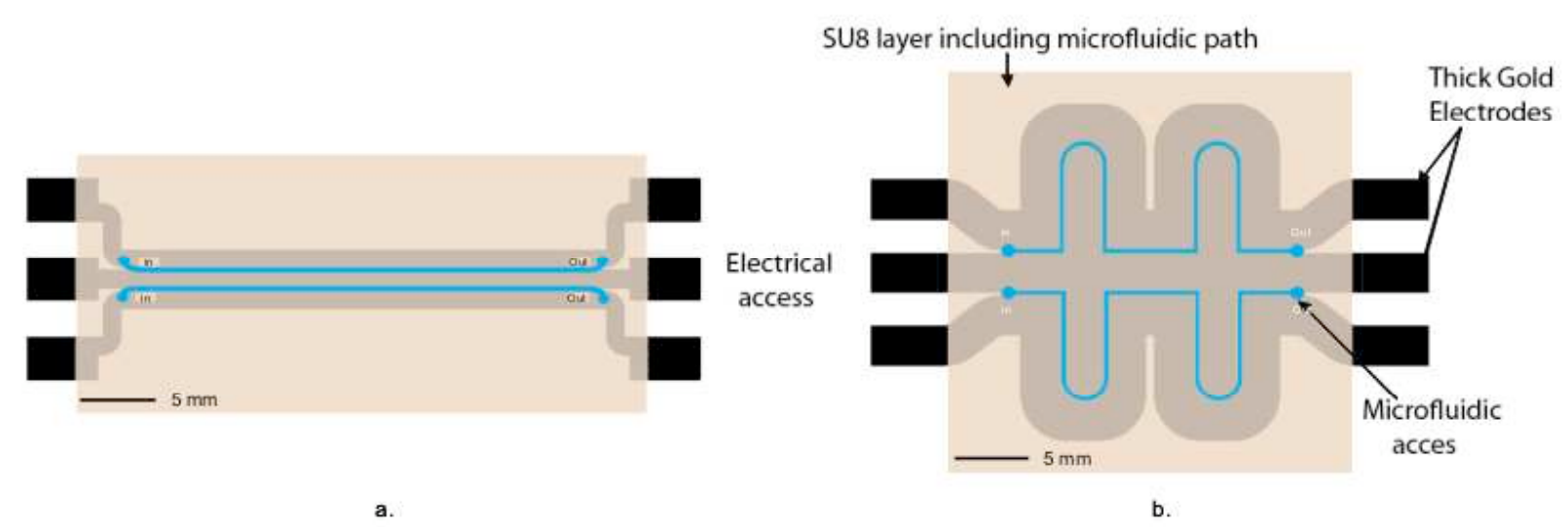

Fig. 2. Top view of the two proposed biochip designs. 
Surface : Electric field, nom [kV/cm]

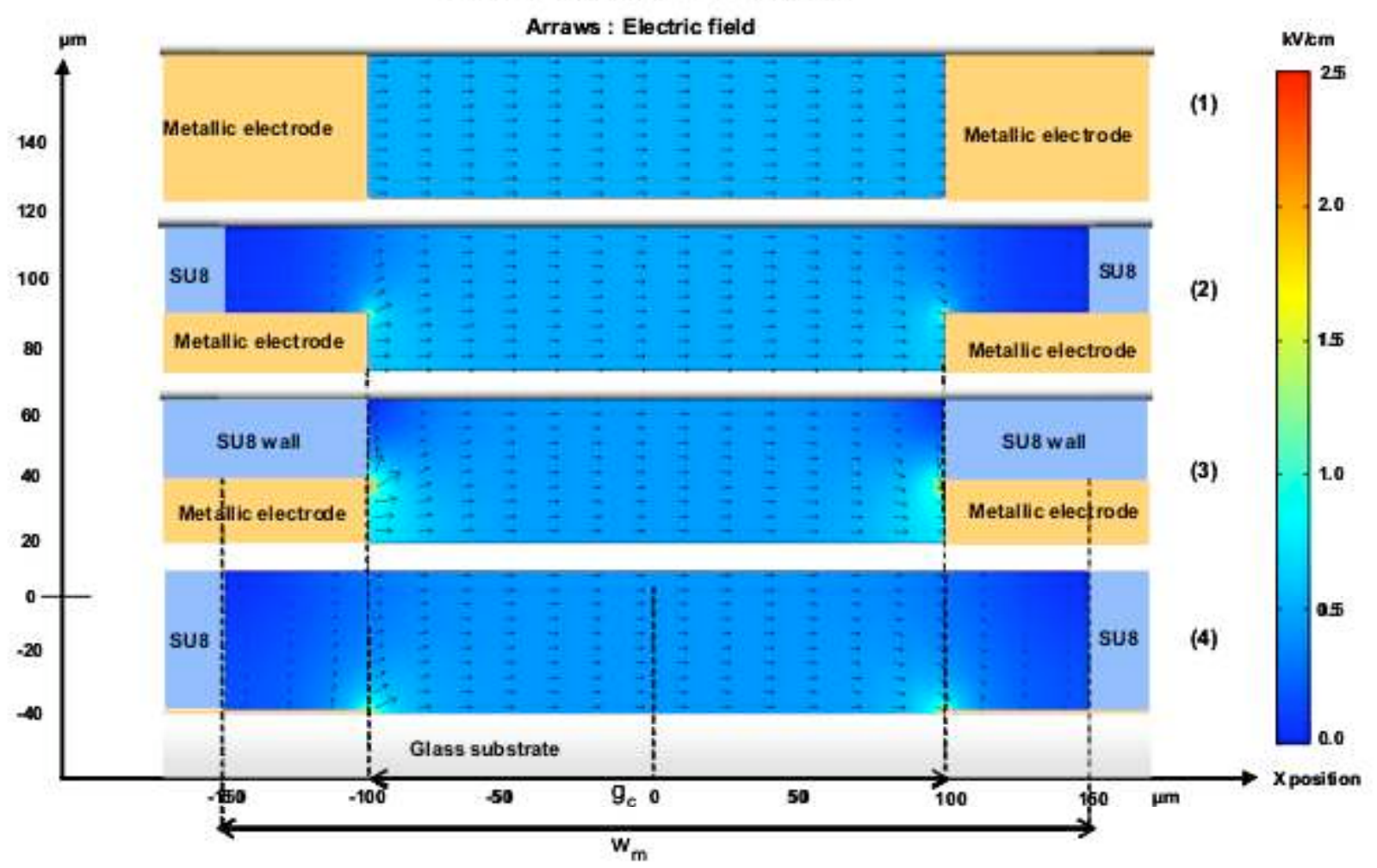

a.

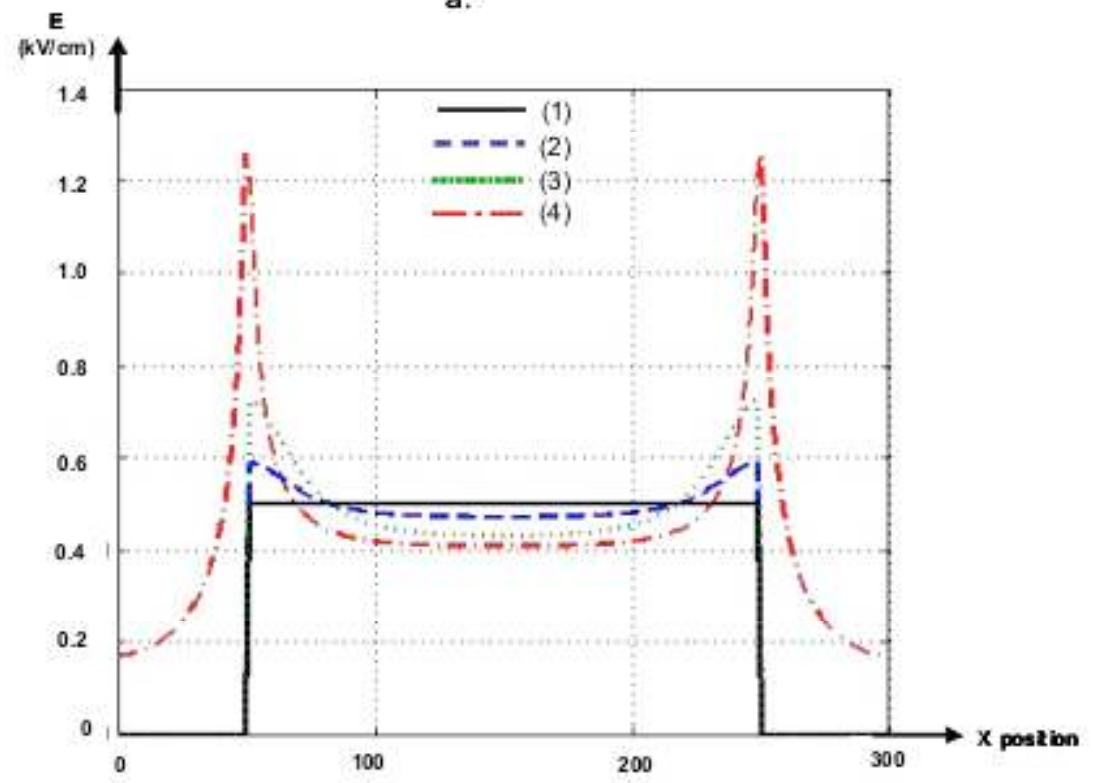

b.

Fig. 3. (a) Simulation of the electric field in the microfluidic channel (cross section). (1) electrodes (thickness eel) define the microchannel height hc, (2) electrodes are thinner (eel $=20 \mu \mathrm{m})$, and covered with SU8 in order to define the whole height of the microchannel (hc=50 $\mu \mathrm{m})$, a recess is introduced for the SU8 channel which becomes larger $(\mathrm{wm}=300 \mu \mathrm{m})$ than the gap between electrodes, (3) case (2) without the recess of the SU8, (4) case (2) with thin electrodes. (b) Plot of the electrical field level along the x position in the microfluidic channel. The electrical field level is plot along a reference line at $3 \mu \mathrm{m}$ above the susbtrate. 


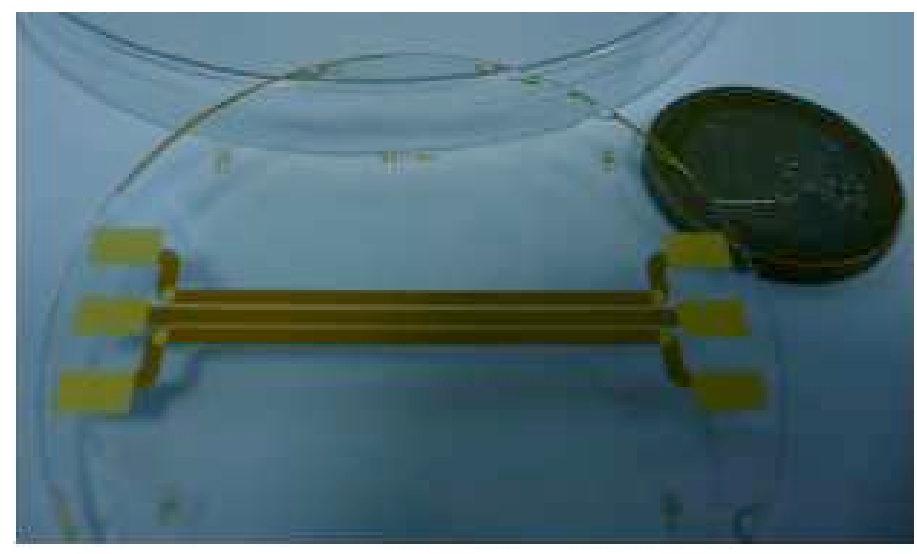

a.

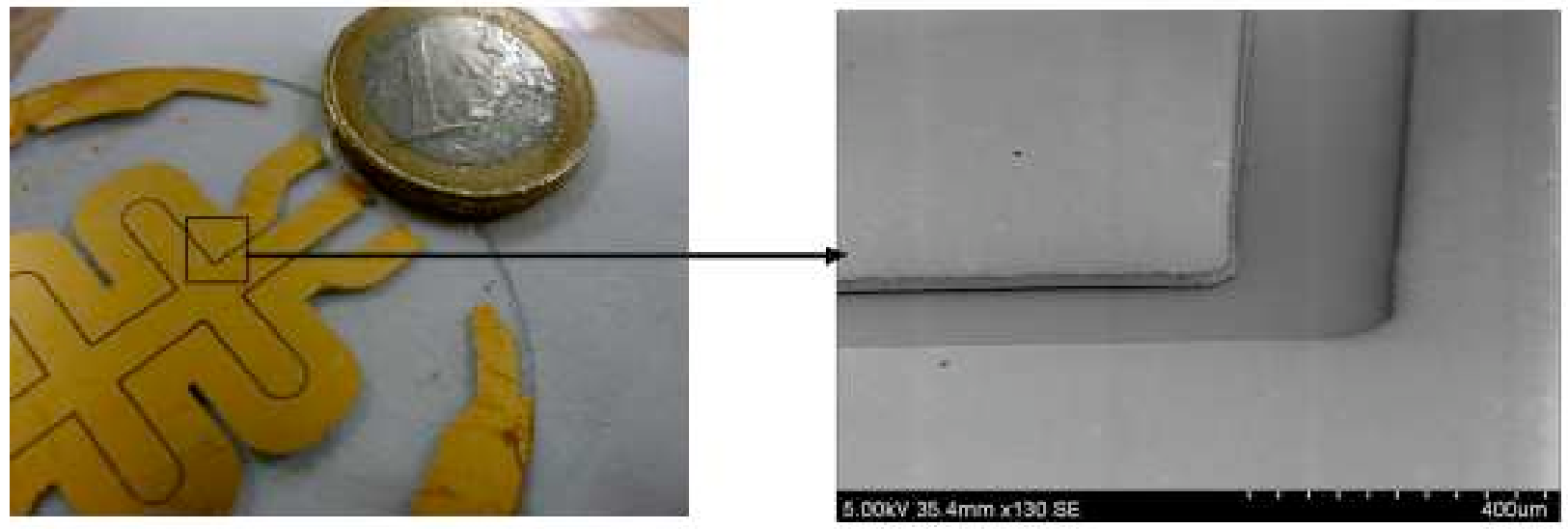

b.

Fig. 4. Photographs of fabricated biochip : (a) case of design 1 - (b) Case of design 2 with magnified SEM view (of the squared zone on figure 4b).
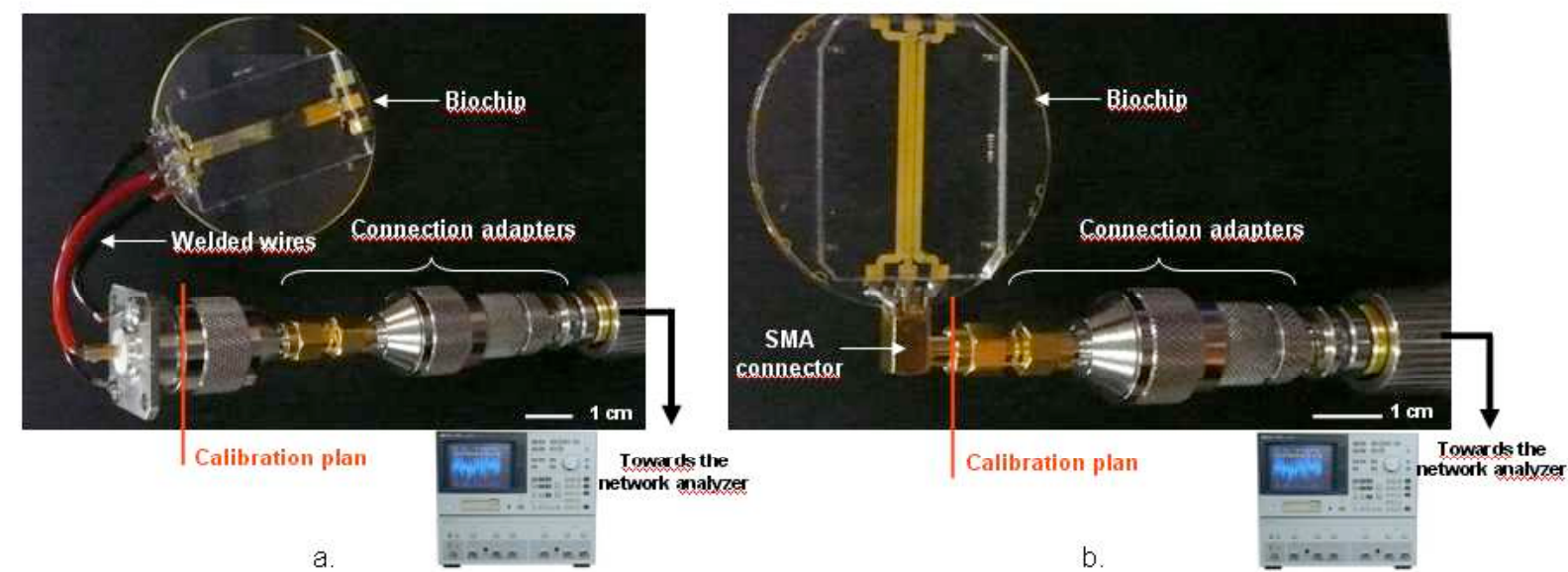

Fig. 5. Photographs of the two different configurations for the biochip connection and setup used for the impedance measurement: (a) with short welded wires - (b) with SMA connectors. 


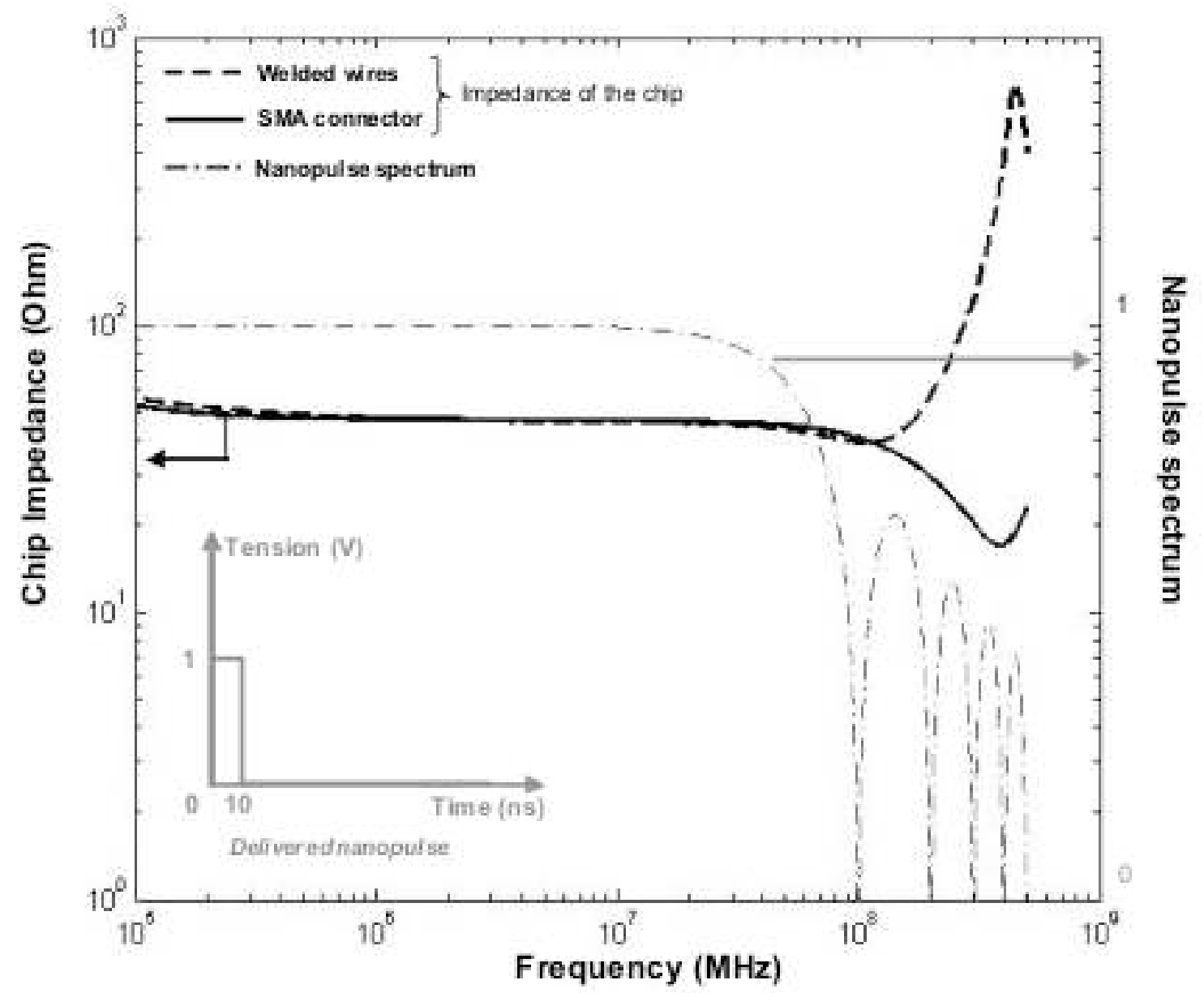

Fig. 6. Comparison between the biochip impedance connected with welded wires (dotted line) and SMA connectors (solid line). Normalized spectrum of a 10 ns duration pulse. 


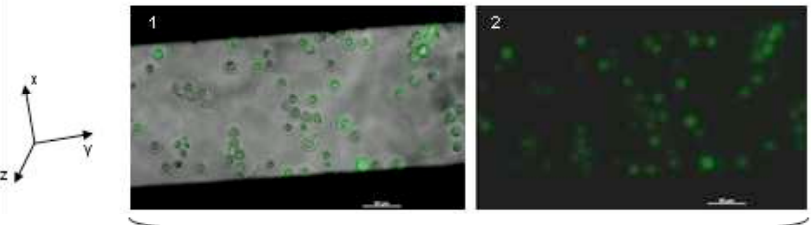

Letcre asper exncsupe

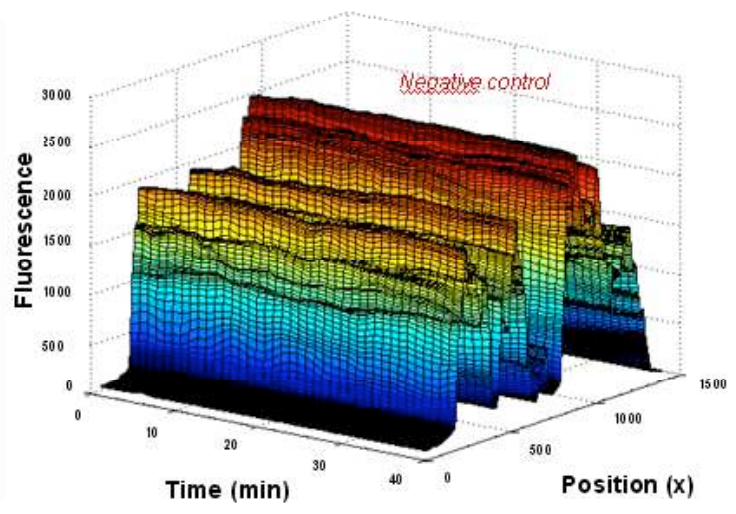

b.

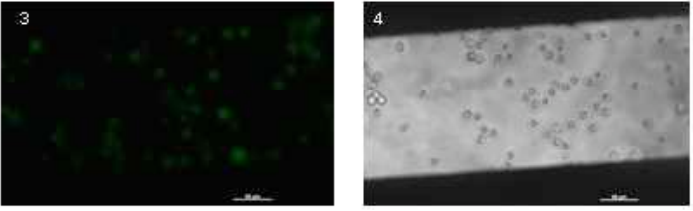

math Taneg she 40 min aher aspeffexnosuc

Finorescence inatoing a.

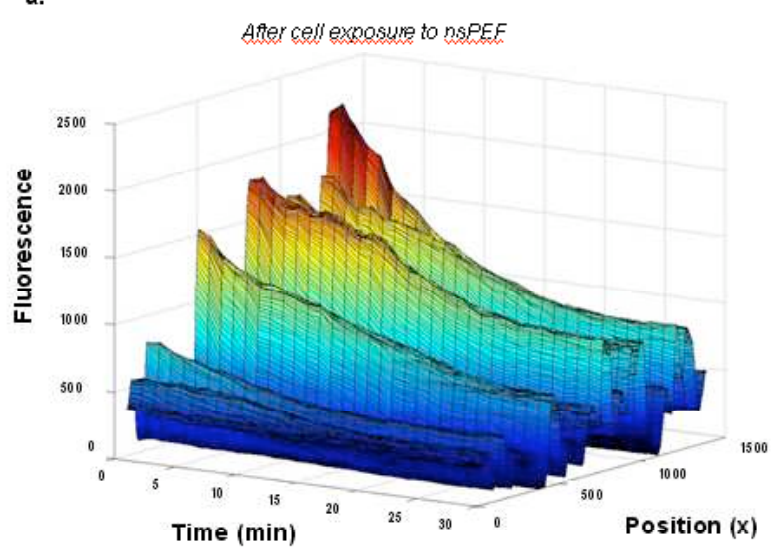

c.

Fig. 7. (a) Monitoring of cells in the microfluidic channel: 1. before nsPEF exposure - Bright field imaging superposed to fluorescence measurement, 2. before nsPEF exposure - Fluorescence imaging of intracellular calcein initial concentration, 3. 20 min after nsPEF exposure (700 $\mathrm{V}$ amplitude) - Fluorecence imaging of intracellular calcein (to be compared with 2.) and 4. $40 \mathrm{~min}$ after nsPEF exposure - Trypan blue experiment (bright field imaging) - (b) Without nsPEF exposure (negative control) - 3D plot of fluorescence level versus time and position along the $\mathrm{x}$ axis - (c) $700 \mathrm{~V}$ amplitude nsPEF exposure (real time monitoring of the fluorecence decay) - 3D plot of fluorecence level versus time and position along the $\mathrm{x}$ axis. 


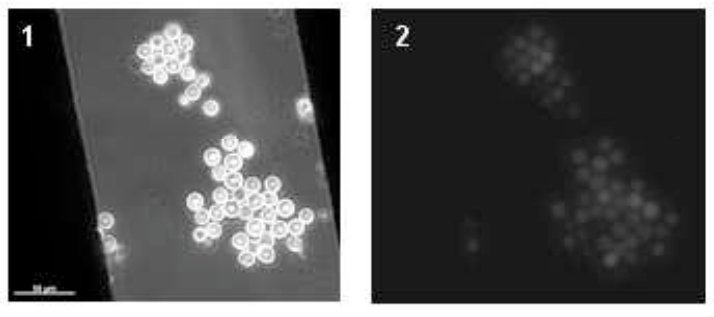

Betore nspef exnosure

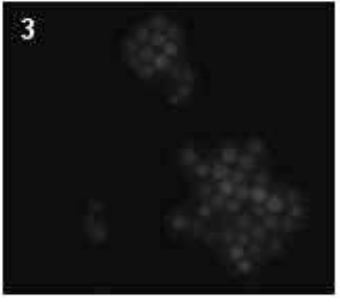

20 min atter nspef exnosure

a.

Affer cell exposure to nsPEF

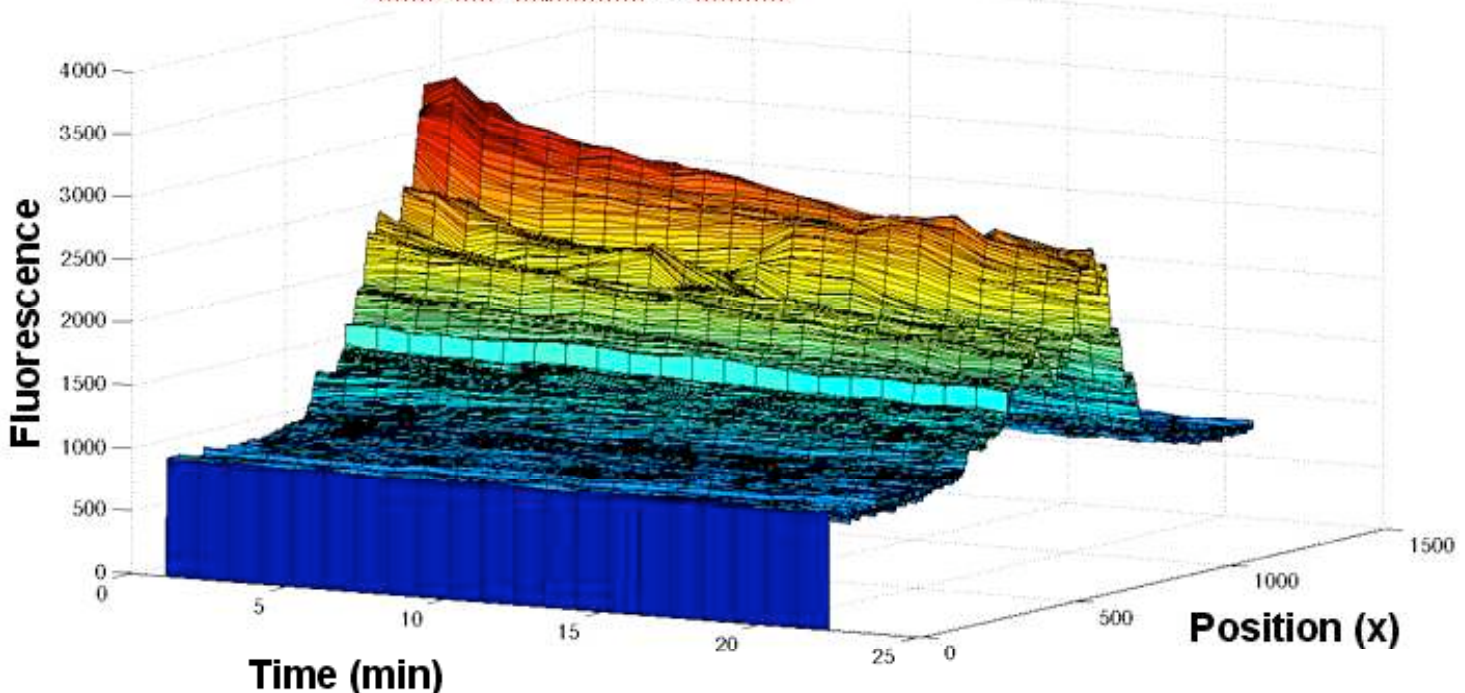

b.

Fig. 8. (a) Monitoring of cells in the microfluidic channel: 1. before nsPEF exposure - Bright field imaging superposed to fluorecence measurement, 2. before nsPEF exposure - Fluorescence imaging of intracellular calcein initial concentration, 3. 20 min after nsPEF exposure (350 $\mathrm{V}$ amplitude) - Fluorecence imaging of intracellular calcein (to be compared with 2.)- (b) $350 \mathrm{~V}$ amplitude nsPEF exposure (real time monitoring of the fluorecence decay) - 3D plot of fluorescence level versus time and position along the $\mathrm{x}$ axis. 
